\title{
Climate of utopia
}

\author{
M. Gedalin ${ }^{1}$ and M. Balikhin ${ }^{2}$ \\ ${ }^{1}$ Department of Physics, Ben-Gurion University, Beer-Sheva, Israel \\ ${ }^{2}$ ACSE, University of Sheffield, Sheffield, UK
}

Received: 7 April 2008 - Revised: 27 May 2008 - Accepted: 30 May 2008 - Published: 7 July 2008

\begin{abstract}
Climate is usually considered to depend on a large number of parameters, this being essentially a functional in multi-dimensional parameter space. We propose a low-dimensional model of a climate where the temperature field on a thermally conducting planet depends on the external energy input and very limited number of internal parameters, like thermal conductivity and reflectivity. Equilibrium temperature and quasistatic variations of climate following slow variations of the energy input are studied. The single phase model exhibits adiabatic behavior and stability with respect to small axisymmetric perturbation. The two phase model shows a non-trivial response to the variations of the external parameter. History dependence, global instabilities and hysteresis behavior characterize the surface temperature evolution.
\end{abstract}

\section{Introduction}

In a non-orthodox way we define climate as a thermal state of a planet which is described by a number of suitable parameters (temperature in the simplest case) and varying at the time scale of larger than that of the seasons. Climate is determined by the external energy input (solar irradiance) as well as the internal parameters (like. e.g., absorption coefficients) and the processes of heat transfer. Heat transfer may be a result of a number of micro-processes like diffusion, material motion, chemical reactions, etc. These are called here microprocesses since they do not necessarily occur at the scale of the whole planet or at the time scale of the climate variations, but may operate at much smaller spatial and temporal scales. When speaking about climate we shall refer to the results of the proper averaging over these micro-processes. Climate

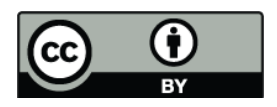

Correspondence to: $\mathrm{M}$. Gedalin (gedalin@bgu.ac.il) variations can occur due to the changes in the solar irradiance (external forcing agent) and/or changes of the internal planetary parameters, like atmosphere composition (internal forcing agent). The large number of possible forcing agents brings about the necessity of sophisticated numerical analyses (see, e.g. Hansen et al., 2005, and references therein). Lower dimensional approaches, which sometimes allow analytical treatment, are based on the globally-averaged energy budget analysis (Boer et al., 2005) or box models (Cai, 2005). Models of intermediate complexity (Petoukhov et al., 2000; Claussen et al., 2002) are particularly attractive since they incorporate a reduced number of micro-processes and/or operate at a reduced level of detail for the sake of simulating the interaction between as many components of the system as possible. Except for the very low-dimensional and conceptual models, analytical study is not possible, and time consuming numerical simulations are necessary. Although sophisticated models are, in a whole, quite successive in the description of the present Earth climate there is a risk that this agreement with observations might be partly a result of tuning (Räisänen, 2007). Long-term predictions are still not sufficiently certain.

The Earth climate varies at large time scales (kyears) in a non-adiabatic manner. Slow changes of the temperature are followed by abrupt climate changes (Alley et al., 2003), like the Dansgaard-Oeschger osciilations (Dansgaard et al., 1982; Rahmstorf, 2001). These oscillations are clear evidence of the nonlinear dynamics of the system, possibly indicating two or more (meta)stable equilibria (Stommel, 1961). A simple conceptual model has been proposed recently (Braun et al., 2007), which postulates the existence of two climate states and a threshold process. The two most central factors affecting the climate variation in such systems are, in our opinion, the oceanic heat content and hear transfer (Rahmstorf, 2001, 2002) and the solar forcing (Van Geel, 1999; Paillard, 2001; Braun et al., 2005; Swindles et al., 2007) (the latter is believed to be due to the Milankovich cycles of the

Published by Copernicus Publications on behalf of the European Geosciences Union and the American Geophysical Union. 
Earths orbit). Numerical simulations in coupled ice-sheet models have been used for studies of glacial climate variations (Oerlemans, 1982; Pollard, 1983; Galleé et al., 1991; Ganopolski et al., 1998; Ganopolski and Rahmstorf, 2001)

In the present paper we suggest a low-dimensional approach, which is based on the ideas similar to those presented by, e.g., Boer et al. (2005) and Cai (2005), developed in the spirit of the nonlinear dynamics models (like Lorentz, 1963). Namely, we suggest that it makes direct physical sense to start building simplified global climate models which would allow mapping of, e.g., temperature distribution on the planet surface, as a function of a small number of parameters. Such a model would incorporate only a very limited number of most important processes, with the objective to establish whether climate is intrinsically stable and what is the influence of the perturbations in the external forcing or the effects of slow (or rapid) changes of the planet parameters (like albedo, thermal conductivity, etc.). In the present paper we propose a simplest model of this kind, where the external forcing is due to the solar heat flux, which is balanced by the thermal black body radiation from the planet. The only modification of the temperature distribution is due to the uniform diffusion-like heat transfer. We study the steady climate and the response to the perturbations of the external forcing. Our objectives are rather modest, and we do not attempt to apply the results of our analysis directly to the interpretation of the Earth climate variations. Instead, we are aiming on establishing the most general tendencies of the climate evolution as depending on a small number of agents, without further complication introduced by multitude of possible micro-processes.

The proposed model is similar to the energy-balance models proposed first by Budyko (1969) and Sellers (1969) and extensively studied during last several decades (see reviews and references in North et al., 1981; North and Stevens, 2006). In these models some terms on the energy balance equations were chosen on a phenomenological basis, and certain boundary conditions were imposed following experimentally established values rather than from the physics of the interaction. We, instead, specify a small number of physical processes governing the system behavior. Accordingly, the boundary and interface conditions are derived from the physics of interaction and not imposed as an external constraint. Thus, physical conclusions derived from the model should be applicable to systems with a wide range of parameters.

\section{Single-phase model}

In this section we develop our model for a single-phase planet. Analysis of the two-phase system is delayed until Sect. 3.

\subsection{The basic model}

We consider an imaginary spherical planet without atmosphere, covered with a thermally conductive layer of a constant width. In order to avoid unnecessary complications, the planet is assumed to be rotating with the rotation axis perpendicular to the direction to the Sun. We shall describe the planet climate with the field of the single scalar variable, daily average temperature, $T(\theta)$, where $\theta$ is the polar angle from the north pole. By doing so we ignore the daynight variations of the temperature which occur on a much faster time scale than we are interested in. A number of processes and parameters which affect the temperature distribution on this planet is small. Let us consider a ring between $\theta$ and $\theta+d \theta$, with the surface area $d S=2 \pi R^{2} \sin \theta d \theta$. Let the temperature of the area change by $d T$ during time $d t$. The heat amount the ring gets is $d Q=c d S d T$, where $c$ is the specific heat of the unit area. This heat comes from the Sun illumination $d Q_{1}=f \sin \theta d S$, where $f$ is the flux density of the solar energy, and $\sin \theta$ takes into account that only the normal component of the flux is absorbed. The (uniform) albedo of the planet surface is included in the definition of the flux $f$. Another contribution is due to the thermal conductivity. Let the heat flux density be $j=(d Q / d L)=-\kappa \nabla T=-(\kappa / R)(d T / d \theta)$. The total heat flux out of the ring would be

$$
\frac{d Q}{d t}=-\frac{\kappa}{R} \frac{d}{d \theta}\left(2 \pi R \sin \theta \frac{d T}{d \theta}\right) d \theta,
$$

so that the total heat gain of the ring area is

$d Q_{g}=\left[f \sin \theta+\frac{\kappa}{R^{2} \sin \theta} \frac{d}{d \theta} \sin \theta \frac{d T}{d \theta}\right] d S d t$.

On the other hand, the amount $d Q_{d}=\sigma T^{4} d S d t$ is dissipated into radiation. Strictly speaking, the radiative term should be $\propto\left\langle T^{4}\right\rangle \neq\langle T\rangle^{4}$, where the angular brackets denote averaging over the planet rotation. For our present purposes the difference may be ignored since the most important feature is that the radiative losses are a nonlinear monotonically increasing function of the daily average temperature. Energy conservation implies $d Q=d Q_{1}-d Q_{2}$, so that

$$
\begin{aligned}
c \frac{\partial T}{\partial t} & =f \sin \theta+k \frac{1}{\sin \theta} \frac{\partial}{\partial \theta} \sin \theta \frac{\partial}{\partial \theta} T-\sigma T^{4}= \\
& =f \sqrt{1-x^{2}}+k \frac{\partial}{\partial x}\left(1-x^{2}\right) \frac{\partial}{\partial x} T-\sigma T^{4},
\end{aligned}
$$

where $x=\cos \theta,-1 \leq x \leq 1$, and $k=\kappa / R^{2}$.

Polar regions require special treatment. For $\theta \rightarrow 0$ the radiative losses are $\propto \theta^{2}$, the incoming flux is $\propto \theta^{3}$ (see, however, below), and the heat flux is $\propto \theta(\partial T / \partial \theta)$. Therefore, one has

$\theta \frac{\partial T}{\partial \theta} \propto \theta^{2} \Rightarrow T \propto \theta^{2}$

In other words, the boundary condition at the poles is

$$
\frac{1}{\theta} \frac{\partial T}{\partial \theta}<\infty
$$


The developed model should be compared to the classical energy-balance models (Budyko, 1969; Sellers, 1969; North et al., 1981) where the black-body radiative term is substituted by a phenomenological radiative term which depends linearly on the temperature. As a result, nonlinearity disappears completely from the model.

We can further reduce the number of parameters by making transformation

$T \rightarrow(k / \sigma)^{1 / 3} T, \quad t \rightarrow(c / k) t$

so that Eq. (4) reduces to the following one-parametric equation

$\frac{\partial T}{\partial t}=F\left(1-x^{2}\right)^{1 / 2}+\frac{\partial}{\partial x}\left(1-x^{2}\right) \frac{\partial}{\partial x} T-T^{4}$,

where $F=f \sigma^{1 / 3} / k^{4 / 3}$. This representation is useful for the analysis of the perturbations of the forcing $f$.

\subsection{Equilibrium}

In the equilibrium temperature is time independent, so that one has

$f\left(1-x^{2}\right)^{1 / 2}+k \frac{d}{d x}\left(1-x^{2}\right) \frac{d}{d x} T-\sigma T^{4}=0$,

or

$f \sin \theta+k \frac{1}{\sin \theta} \frac{d}{d \theta} \sin \theta \frac{d}{d \theta} T-\sigma T^{4}=0$.

The first term in this equation is the energy input from outside, the second one is the energy transfer to the poles, while the last one is the dissipation. If $f=0$ the only solution is the trivial one, $T=0$. If $\sigma=0$ there is no equilibrium solution. If $k=0$ the obvious solution is $T=(f \sin \theta / \sigma)^{1 / 4}$, so that the temperature on the poles is zero, while the maximum (equatorial) temperature is $T_{0}=(f / \sigma)^{1 / 4}$ It is easy to see that when $k \neq 0$, the temperature on the poles cannot vanish since the nonzero energy flux into the pole region should be balanced by the radiation. Near the poles, $\theta \ll 1$, Eq. (10) can be approximately written as

$f \theta+k \frac{1}{\theta} \frac{d}{d \theta} \theta \frac{d}{d \theta} T-\sigma T^{4}=0$

We shall seek for a solution of the form

$T=\tilde{T}_{0}+A_{1} \theta^{\alpha}+A_{2} \theta^{\alpha+1}+\ldots$,

where $\alpha>0$. Direct substitution immediately gives $\alpha=2$ and

$T=\tilde{T}_{0}+\left(\sigma \tilde{T}_{0}^{4} / 4 k\right) \theta^{2}-(f / 9 k) \theta^{3}+\ldots$

For $k \rightarrow \infty$ one has $d T / d \theta=0$ and $T=T_{a v}=$ const (other solutions diverge) and, therefore,

$4 \pi \sigma T_{a v}^{4}=2 \pi \int_{0}^{\pi} f \sin ^{2} \theta d \theta=\pi^{2} f$

and $T_{a v}=T_{0}(\pi / 4)^{1 / 4}$.

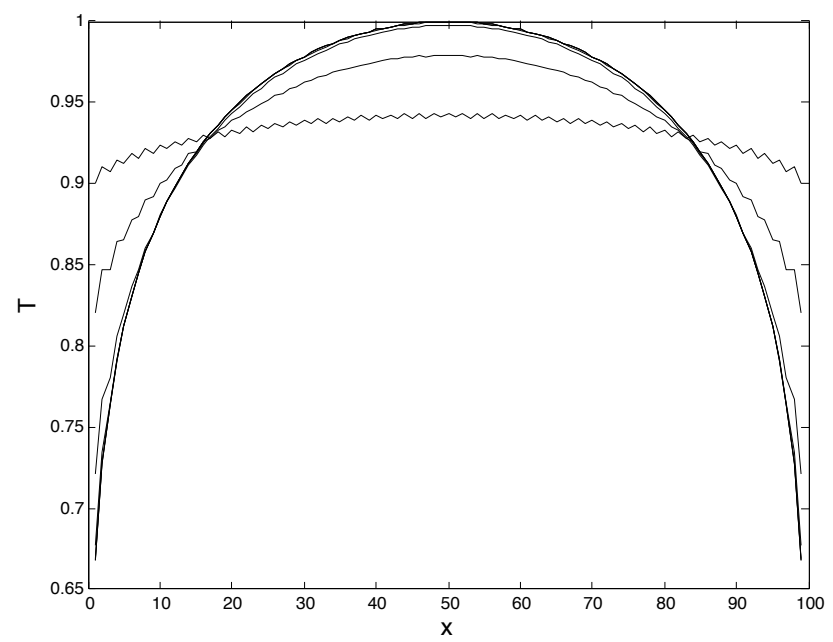

Fig. 1. Temperature distribution as a function of $x=\cos \theta$ for various $K=0.01,0.1,1,10,100$.

In general, it makes sense to normalize the temperature as follows: $\tilde{T}=T / T_{0}=T \sigma^{1 / 4} f^{-1 / 4}$, so that Eq. (9) is rewritten in the form

$\left(1-x^{2}\right)^{1 / 2}+K \frac{\partial}{\partial x}\left(1-x^{2}\right) \frac{\partial}{\partial x} \tilde{T}-\tilde{T}^{4}=0$,

where $K=k / \sigma T_{0}^{3}=k \sigma^{-1 / 4} f^{-3 / 4}$.

Figure 1 shows distribution of the normalized temperature $\tilde{T}$ as a function of $x=\cos \theta$ for various values of the parameter $K=0.01,0.1,1,10,100$. Higher $K$ correspond to higher heat conductivity, and, therefore, to the more homogeneous temperature distributions.

\subsection{Perturbation analysis}

Let us assume that we succeeded to find an equilibrium solution $T_{e q}(\theta)$. It it is perturbed, $T=T_{e q}+T_{1}, T_{1} \ll T_{e q}$, the equation for the perturbation takes the following form:

$\frac{\partial T_{1}}{\partial t}=K \frac{1}{\sin \theta} \frac{\partial}{\partial \theta} \sin \theta \frac{\partial}{\partial \theta} T_{1}-4 T_{e q}^{3} T_{1}$,

where $t /\left(c / \sigma T_{0}^{3}\right) \rightarrow t$. Putting $T_{1} \propto \exp (p t)$, we have the equation for the eigenvalue problem:

$L T_{1}=p T_{1}$,

where

$$
\begin{aligned}
& L=K \frac{1}{\sin \theta} \frac{\partial}{\partial \theta} \sin \theta \frac{\partial}{\partial \theta}-4 T_{e q}^{3} \\
& =K \frac{\partial}{\partial x}\left(1-x^{2}\right) \frac{\partial}{\partial x}-4 T_{e q}^{3}
\end{aligned}
$$


It is easy to find $(*$ denotes complex conjugate)

$$
\begin{aligned}
& T_{1}^{*} L T_{1}+T_{1} L T_{1}^{*}=K \frac{\partial}{\partial x}\left[\left(1-x^{2}\right) \frac{\partial}{\partial x}\left|T_{1}\right|^{2}\right] \\
& -K\left(1-x^{2}\right)\left|\frac{\partial T_{1}}{\partial x}\right|^{2}-4 T_{e q}^{3}\left|T_{1}\right|^{2}=2 \operatorname{Re} p\left|T_{1}\right|^{2}
\end{aligned}
$$

Integrating over $-1 \leq x \leq 1$ one easily gets $\operatorname{Re} p<0$, which means that all perturbations damp.

In the limit of zero thermal conductivity $K=0$ and one immediately has

$T_{e q}=\sin ^{1 / 4} \theta$

Respectively, Eq. (17) is immediately solved

$T_{1} \propto \exp \left(-4 T_{e q}^{3} t\right)$

In the limit of the very high thermal conductivity, $K \gg 1$, one has $T_{e q}=T_{a v}=$ const, so that the equation for the perturbation reads

$K \frac{\partial}{\partial x}\left(1-x^{2}\right) \frac{\partial T_{1}}{\partial x}=\left(p+4 T_{a v}^{3}\right) T_{1}$.

It has the solutions in the form of Legendre polynomials with $\left(p+4 T_{a v}^{3}\right) / K=-n(n+1), n \geq 0$ and integer. The smallest damping rate is achieved for $n=0$ and is $p=-4 T_{e q}^{3}$, as above.

\subsection{Perturbed forcing}

Let us now consider the response of the system to small forcing perturbations. In other words, let $T_{S}(x)$ be the solution of the stationary Eq. (9) and we seek for the solution of the time-dependent equation

$$
\frac{\partial T}{\partial t}=(1+\epsilon \eta(t))\left(1-x^{2}\right)^{1 / 2}+K \frac{\partial}{\partial x}\left(1-x^{2}\right) \frac{\partial}{\partial x} T-T^{4},
$$

where $\epsilon$ is the smallness parameter. Assuming $T=T_{e q}+\epsilon T^{\prime}$, one gets

$$
\frac{\partial T^{\prime}}{\partial t}-K \frac{\partial}{\partial x}\left(1-x^{2}\right) \frac{\partial}{\partial x} T^{\prime}+4 T_{e q}^{3} T^{\prime}=\eta(t)\left(1-x^{2}\right)^{1 / 2} .
$$

Since this equation is linear, one may put $\eta(t)=\eta_{0} \exp (-i \omega t)$, and $T^{\prime}=\tilde{T} \exp (-i \omega t)$. One immediately gets

$$
-i \omega T^{\prime}=L T^{\prime}+\eta_{0}\left(1-x^{2}\right)^{1 / 2} .
$$

Expanding $T^{\prime}$ in terms of orthonormal eigenfunctions $L T_{p}=p T_{p}, T^{\prime}=\sum_{p} \alpha_{p} T_{p}$, one has

$\alpha_{p}=-\frac{\eta_{0}}{p+i \omega} \int_{-1}^{1}\left(1-x^{2}\right)^{3 / 2} T_{p} d x$

Since $p$ is real, the response is always nonresonant.

\section{Two-phase model description}

In this section we extend the analysis of the model developed in Sect. 2.1 onto a two-phase system. Our attention will be mainly devoted to the equilibrium dependence on the planet parameters as well as the response of the system to slow external variations.

\subsection{The two-phase model}

We assume that the substance covering the surface of the planet can exist in two phases: a) "ice" at temperatures $T \leq T_{c}$ (subscript "1"), and b) "water" at temperatures $T \geq T_{c}$ (subscript "2"). Each phase is characterized by its own specific heat $c_{i}$ (per unit area). The latent (melting) heat is $\lambda$ per unit area. We shall also assume that different phases absorb radiation differently, the coefficient $\alpha_{i}$ is the fraction of the incident flux which is absorbed (1- $\alpha$ is reflected). For each phase the equation for the local temperature reads

$c_{i} \frac{\partial T_{i}}{\partial t}=\alpha_{i} f \sin \theta+k_{i} \frac{1}{\sin \theta} \frac{\partial}{\partial \theta} \sin \theta \frac{\partial}{\partial \theta} T_{i}-\sigma T_{i}^{4}$

where $k_{i}$ are the properly normalized specific thermal conductivities. If the temperature everywhere is too low, $T<T_{c}$ for all $\theta$, the planet is covered be ice. If $T>T_{c}$ for all $\theta$ the planet is covered by water. In both cases the single phase model is applicable.

If there are regions with $T=T_{c}$ a mixed state is possible. We shall assume that such mixed can be represented as two icy polar caps, $0 \leq \theta<\theta_{p}$ and $\pi-\theta_{p}<\theta \leq \pi$, and water between them, $\theta_{p}<\theta<\pi-\theta_{p}$. In what follows we shall restrict our analysis with one hemisphere $0 \leq \theta \leq \pi / 2$, because of the symmetry. In each region, icy or watery, the temperature distribution is described the Eq. (27).

At the boundary $\theta=\theta_{p}$ the temperatures should be equal to the melting temperature, $T_{i}=T_{c}$, unless heat transfer between ice and water is inhibited (see below).

In general, the boundary may be moving, $\dot{\theta}_{p} \neq 0$. Let the ice-water boundary move by $\delta \theta_{p}=\dot{\theta}_{p} d t$ toward the equatorial plane. The amount of energy added to the ring with the radius $R \sin \theta_{p}$ and the width $R \delta \theta_{p}$ is $\delta Q=-\lambda d S=-2 \pi R^{2} \lambda \sin \theta_{p} \dot{\theta}_{p} d t$, where $\lambda$ is the specific latent heat. This energy is due to the difference between the fluxes from the both sides of the boundary

$\delta Q=\left[-k_{1}{\frac{\partial T_{1}}{\partial \theta}}_{\theta=\theta_{p}}+k_{2}{\frac{\partial T_{2}}{\partial \theta}}_{\theta=\theta_{p}}\right] 2 \pi R \sin \theta_{p} d t$

which gives

$\lambda R \dot{\theta}_{p}=-\left[k_{2} \frac{\partial}{\partial \theta} T_{2}-k_{1} \frac{\partial}{\partial \theta} T_{1}\right]_{\theta=\theta p}$

It is worth noting that the change of the absorbed power $\delta P^{\prime}=\left(\alpha_{1}-\alpha_{2}\right) f \sin \theta_{p}\left(2 \pi R^{2} \sin \theta_{p}\right) \cdot \theta_{p} d t$ gives the energy 
change only in the second order, $\delta Q^{\prime} \sim \delta P^{\prime} \delta t$. In the stationary state $\dot{\theta}_{p}=0$ and

$\left[k_{2} \frac{\partial}{\partial \theta} T_{2}-k_{1} \frac{\partial}{\partial \theta} T_{1}\right]_{\theta=\theta_{p}}=0$

It is instructive to arrive at the equation for the boundary motion from the consideration of the "total heat content"

$\frac{Q_{c}}{2 \pi R^{2}}=\int_{0}^{\theta_{p}} c_{1} T_{1} \sin \theta d \theta$
$+\int_{\theta_{p}}^{\pi / 2}\left(c_{1} T_{c}+c_{2}\left(T_{2}-T_{c}\right)+\lambda\right) \sin \theta d \theta$

where $\quad T_{1}=T_{1}(\theta), \quad T_{2}=T_{2}(\theta), \quad \theta_{p}=\theta_{p}(t), \quad$ and $T_{1}\left(\theta_{p}\right)=T_{2}\left(\theta_{p}\right)=T_{c}$. Here $\lambda$ is the latent heat necessary to melt the ice to water at the melting temperature $T_{c}$. The heat content change rate due to solar irradiance and radiative losses is

$$
\begin{aligned}
\frac{\dot{Q}_{r}}{2 \pi R^{2}} & =\alpha_{1} f \int_{0}^{\theta_{p}} \sin ^{2} \theta d \theta+\alpha_{2} f \int_{\theta_{p}}^{\pi / 2} \sin ^{2} \theta d \theta \\
& -\sigma \int_{0}^{\theta_{p}} T_{1}^{4} \sin \theta d \theta-\sigma \int_{\theta_{p}}^{\pi / 2} T_{2}^{4} \sin \theta d \theta \\
& =\frac{f}{4}\left[\alpha_{1} \pi+\left(\alpha_{2}-\alpha_{1}\right)\left(\pi-2 \theta_{p}+\sin 2 \theta_{p}\right)\right] \\
& -\sigma \int_{0}^{\theta_{p}} T_{1}^{4} \sin \theta d \theta-\sigma \int_{\theta_{p}}^{\pi / 2} T_{2}^{4} \sin \theta d \theta
\end{aligned}
$$

Energy conservation requires (in what follows we omit the constant non-essential factor $2 \pi R^{2}$ )

$\dot{Q}_{c}=\dot{Q}_{r}$

Using Eq. (27) one arrives again to Eq. (28).

Additional boundary conditions read

$\frac{1}{\sin \theta} \frac{\partial T_{1}}{\partial \theta} \rightarrow 0, \quad \theta \rightarrow 0$,

$\frac{\partial T_{2}}{\partial \theta}=0, \quad \theta=\pi / 2$

the first of which have been obtained earlier while the second follows from the symmetry $\theta \leftrightarrow \pi-\theta$.

The derived equations for the temperature evolution together with the condition at the ice-water boundary give a complete description of the system. In what follows we start the analysis of the set with simple limiting cases. We will be mostly interested in stationary states and their perturbations.

It is worth noting that previous energy-balance models (Budyko, 1969; Sellers, 1969; Cahalan and North, 1979; North et al., 1981; North, 1984; North and Stevens, 2006; Wu and North, 2007) treated the two-phase system with the use of the temperature-dependent albedo (and possibly specific heat and thermal conductivity), where the albedo above the critical temperature corresponds to that of the water, and below the critical temperature corresponds to that of the ice. The boundary condition at the ice cap boundary has been imposed phenomenologically (see, e.g. North et al., 1981) by specifying $T\left(\theta_{p}\right)$. The melting-freezing process was not considered. Here we explicitly introduce teh latent heat for the ice melting, and derive the boundary conditions from the first principles.

\subsection{No heat conductance}

In the absence of heat conductance, $k_{i}=0$, the local temperature is determined by the local energy balance,

$c_{i} \frac{\partial T_{i}}{\partial t}=\alpha_{i} f \sin \theta-\sigma T_{i}^{4}$

Absence of the heat flux across the ice-water boundary allows jump of the temperature at the boundary, which, in turn, permits stationary solutions of the form

$T_{i}=\left(\alpha_{i} f \sin \theta / \sigma\right)^{1 / 4}$

Such solution is possible only for $\alpha_{1}<\alpha_{2}$ ("normal" case), since only in this case it is possible to achieve the necessary relation

$$
\begin{aligned}
& T_{1}\left(\theta_{p}\right)=\left(\alpha_{1} f \sin \theta_{p} / \sigma\right)^{1 / 4} \leq T_{c} \\
& \leq\left(\alpha_{2} f \sin \theta_{p} / \sigma\right)^{1 / 4}=T_{2}\left(\theta_{p}\right)
\end{aligned}
$$

The polar cap size $\theta_{p}$ is not determined unambiguously by the equations and may be within the limits restricted by Eq. (38):

$$
\frac{\sigma T_{c}^{4}}{\alpha_{2} f} \leq \sin \theta_{p} \leq \frac{\sigma T_{c}^{4}}{\alpha_{1} f}
$$

provided $\sigma T_{c}^{4} / \alpha_{2} f<1$. When $\sigma T_{c}^{4} / \alpha_{2} f \geq 1$ the whole planet is covered by ice. The opposite (whole planet covered by water) is impossible since the radiation flux is always zero at the pole.

The case $\alpha_{1}>\alpha_{2}$ ("abnormal" case) does not allow stationary solutions, since at the ice-water boundary one would have $T_{1}>T_{2}$ which is impossible.

Let us now consider the adiabatic switch-on of the radiation in the normal case $\alpha_{1}<\alpha_{2}$. For very low $f$ the whole planet is covered with ice. With the increase of $f$ the temperature increases. It first reaches $T=T_{c}$ at the equator, where the ice starts to melt. Once melted the absorbed energy increases so that the temperature jumps to $T_{w}=T_{c}\left(\alpha_{2} / \alpha_{1}\right)^{1 / 4}$. With the further increase of the irradiation the ice-water boundary moves poleward with the ice temperature at the boundary equal to the melting temperature while the water temperature $T_{w}>T_{c}$. If we now start to decrease $f$ adiabatically, the temperature starts to decrease on both sides until water begins to freeze. Eventually the boundary moves toward equator and the water temperature at the boundary is $T_{c}$ while the ice temperature is $T_{i}=T_{c}\left(\alpha_{1} / \alpha_{2}\right)^{1 / 4}<T_{c}$.

In the abnormal case the beginning of melting results in the decrease of energy absorption which should stop the melting. A quasi-stationary state cannot be achieved and a time-dependent state is developing. With the increase of $f$ the planet develops into a three-zone system: a watery zone around the equatorial plane, icy polar caps, and an intermediate zone with time-dependent melting and freezing. We shall not devote more time to the abnormal case here. 


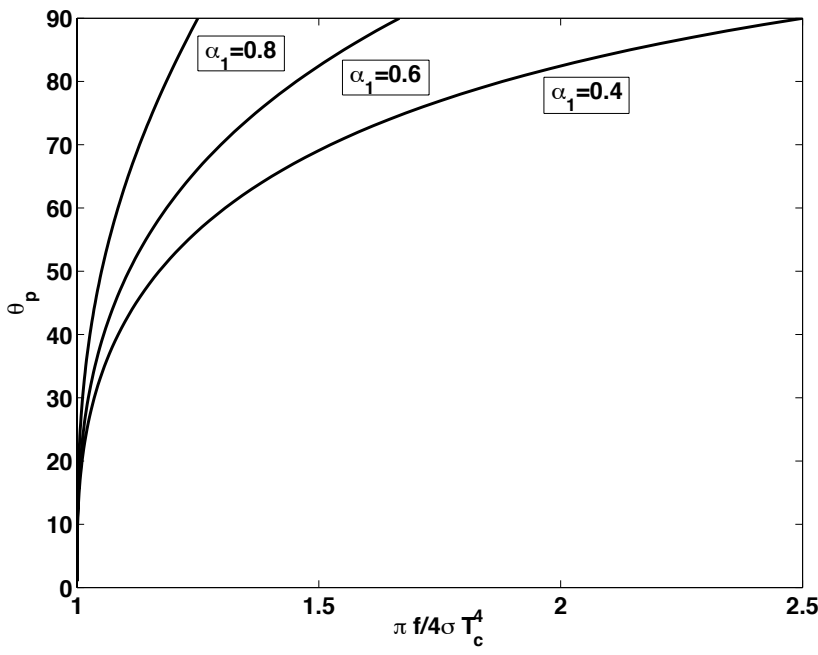

Fig. 2. Dependence of the angular size of the ice cap $\theta_{p}$ on the solar radiation constant $f$ for $\alpha_{1}=0.4,0.6,0.8$, when $\alpha_{2}=1$.

\subsection{Uniform temperature}

If $k_{1}=k_{2}=\infty$ the temperature should be uniform all over the planet. If $T>T_{c}$ then the whole planet is covered with water, if $T<T_{c}$ the planet surface is completely ice. The two phases may coexist if $T=T_{c}$. In this case the total heat content is

$Q_{c}=c_{1} T_{c}+\lambda \cos \theta_{p}$

and the integral energy conservation gives

$$
\begin{aligned}
& -\lambda \sin \theta_{p} \dot{\theta}_{p}=\frac{\pi f}{4}\left[\alpha_{1}\right. \\
& \left.+\left(\alpha_{2}-\alpha_{1}\right)\left(1-2 \theta_{p} / \pi+\sin 2 \theta_{p} / \pi\right)\right]-\sigma T_{c}^{4}
\end{aligned}
$$

Stationary state is achieved when

$$
f\left[1+(s-1) \phi\left(\theta_{p}\right)\right]=f_{1}
$$

where we introduced the notation

$$
\begin{aligned}
& s=\alpha_{2} / \alpha_{1}, \quad f_{1}=4 \sigma T_{c}^{4} / \pi \alpha_{1}, \\
& f_{2}=4 \sigma T_{c}^{4} / \pi \alpha_{2}=f_{1} / s, \\
& \phi(\theta)=1-2 \theta / \pi+\sin 2 \theta / \pi
\end{aligned}
$$

The function $\phi(\theta)$ is a monotonically decreasing function of the angle with $\phi(0)=1$ and $\phi(\pi / 2)=0$. Figure 2 shows the dependence of $\theta_{p}$ on $f$ for several $\alpha_{1}$ (for convenience $\left.\alpha_{2}=1\right)$.

We shall analyze in detail all options. If $T<T_{c}$ the whole planet is covered with ice. In this case $\pi f \alpha_{1} / 4=\sigma T^{4}<\sigma T_{c}^{4}$ which is possible only for $f<f_{1}$. If $T>T_{c}$ the whole planet is covered with water which is possible only if $f>f_{2}$. In the mixed regime $T=T_{c}$ and $\min \left(f_{1}, f_{2}\right)<f<\max \left(f_{1}, f_{2}\right)$. Let us consider separately the normal case $s>1$ and the abnormal case $s<1$.

\subsubsection{Normal case}

In this case $s>1$ and $f_{2}<f_{1}$. The mixed state exists for $f_{2}<f<f_{1}$, and Eq. (42) immediately gives

$\phi=\frac{1}{s-1}\left(f_{1} / f-1\right)$

Since $d \phi / d f<0$ and $d \phi / d \theta_{p}<0$, one has $d \theta_{p} / d f>0$, which means that when the irradiance increases the icy polar cap increases too! This happens because the increase of the polar cap reduces the absorption of the incoming heat which is necessary to maintain the same temperature $T_{c}$ and the balance between the energy income and outcome (the latter remains constant). We show below, however, that this quasistationary behavior is unstable and cannot be observed.

Let us now consider the adiabatic (slow) changes of the heat flux $f$. Let us start with $f<f_{2}$ and $T<T_{c}$ (ice only). Gradual increase of $f$ results in the gradual increase of the temperature until the melting point $T=T_{c} v$ is achieved when $f=f_{1}$. At this point the ice melts completely, the radiation absorption becomes stronger, and the temperature jumps to $T_{w}=T_{c} s^{1 / 4}$. Slow decrease of the flux results in the gradual decrease of the water temperature down to the freezing point at $f=f_{2}$ where the temperature falls to $T_{i}=T_{c} s^{-1 / 4}$. Thus, a history dependent hysteresis behavior of the planet temperature should be observed while the planet should be covered by a single phase (either ice of water), except for the transient periods of melting and freezing. A quasi-stationary mixed state cannot be achieved.

Let us now assume that a mixed state is somehow achieved as an initial condition. A small increase of the flux would the result in melting of a small part of the ice, which would result in the increase of the radiation absorption, further melting, and so on, until all ice would melt and the planet would switch into a single phase. Similar reduction of the flux would result in the freezing of the whole planet. Thus, the mixed state is unstable. This can be shown quantitatively. Let us start with a mixed-state equilibrium, where $f=f_{0}$ and $\theta_{p}=\theta_{0}$ are related by the relation $f_{0}\left[1+(s-1) \phi\left(\theta_{0}\right)\right]=f+1$. Let us consider small perturbations $f=f_{0}+\delta f, \theta_{p}=\theta_{0}+\delta \theta$, then one has

$$
\begin{aligned}
& -\lambda \sin \theta_{0} \dot{\delta} \theta=\sigma T_{c}^{4} \delta f / f_{0}-\mu^{2}(s-1) \delta \theta, \\
& \mu^{2}=-\left(\pi f_{0} \alpha_{1} / 4\right)(d \phi / d \theta)_{\theta=\theta_{0}}
\end{aligned}
$$

It is easily seen that the homogeneous part of the equation has exponentially growing solutions

$\delta \theta \propto \exp (p t), \quad p=\mu^{2}(s-1) / \lambda \sin \theta_{0}$

Equation (47) is easily solved:

$\delta \theta=-\frac{\sigma T_{c}^{4}}{\lambda \sin \theta_{0} f_{0}} \int_{0}^{t} \delta f\left(t^{\prime}\right) e^{p\left(t-t^{\prime}\right)} d t^{\prime}$

If $\delta f^{\prime}$ varies slowly at the time scale $1 / p$ one has

$\delta \theta \approx-\frac{\sigma T_{c}^{4} \delta f}{\mu^{2}(s-1) f_{0}}\left(e^{p t}-1\right)$ 
that is, exponentially fast contraction of the polar cap (for $\delta f>0)$ at the time scale $1 / p$.

\subsubsection{Abnormal case}

In this case $s<1$ and $f_{1}<f_{2}$. As a result, melting starts at lower fluxes, and a stable mixed state exists for $f_{2}<f<f_{1}$, where $d \theta_{p} / d f<0$. The latter means that gradual increase of the radiation flux results in the gradual melting of the ice and gradual contraction of the polar cap. Same analysis as above shows that the state is stable: perturbations decay exponentially and the system falls back into a quasi-stationary regime.

\section{Global stability: general approach}

For finite nonzero values of the thermal conductivity coefficients analysis is more difficult. We are interested here in the global stability: let us assume that the ice-water boundary moves by and infinitesimal displacement $\delta \theta_{p}$ during the infinitesimal time $\delta t$. The energy conservation Eq. (33) gives

$\frac{\delta Q_{c}}{\delta \theta_{p}} \frac{d}{d t} \delta \theta_{p}=\frac{\delta \dot{Q}_{r}}{\delta \theta_{p}} \delta \theta_{p}$.

It is clear that if

$\frac{\delta Q_{c}}{\delta \theta_{p}} \cdot \frac{\delta \dot{Q}_{r}}{\delta \theta_{p}}>0$

the system is unstable since the displacement will exponentially grow.

Since the functional form of the temperature depends on $\theta_{p}$ we shall write $T_{1}=T_{1}\left(\theta, \theta_{p}\right), T_{2}=T_{2}\left(\theta_{p}, \theta\right)$, and denote

$\delta T=\frac{\delta T}{\delta \theta_{p}} \delta \theta_{p}$

Now the variational derivatives can be expressed as follows:

$$
\begin{aligned}
\frac{\delta Q_{c}}{\delta \theta_{p}} & =-\lambda \sin \theta_{p}+\int_{0}^{\theta_{p}} c_{1} \frac{\delta T_{1}}{\delta \theta_{p}} \sin \theta d \theta \\
& +\int_{\theta_{p}}^{\pi / 2} c_{2} \frac{\delta T_{2}}{\delta \theta_{p}} \sin \theta d \theta
\end{aligned}
$$

$$
\begin{aligned}
\frac{\delta \dot{Q}_{r}}{\delta \theta_{p}} & =-f\left(\alpha_{2}-\alpha_{1}\right) \sin ^{2} \theta_{p} \\
& -4 \sigma \int_{0}^{\theta_{p}} T_{1}^{3} \frac{\delta T_{1}}{\delta \theta_{p}} \sin \theta d \theta \\
& -4 \sigma \int_{\theta_{p}}^{\pi / 2} T_{2}^{3} \frac{\delta T_{2}}{\delta \theta_{p}} \sin \theta d \theta
\end{aligned}
$$

Unfortunately, $T_{1}\left(\theta, \theta_{p}\right), T_{2}\left(\theta_{p}, \theta\right)$ cannot (at least, at the present stage) be found explicitly in general case. We shall consider the case of high but finite thermal conductivities.

\subsection{High finite thermal conductivity}

We are unable to solve analytically the set of the Eqs. (27), (28), (34) and (35). However, analysis is possible for high (but finite) thermal conductivities. Indeed, Fig. 1 shows that the temperature profile for large $K$ does not vary much around an average value. We can, therefore, assume that $T_{i}=T_{c}+\tau_{i}$, where $\tau_{i} \propto 1 / k_{i}$. In the stationary case, substituting into Eq. (27), in the lowest nontrivial order one has

$$
\frac{1}{\sin \theta} \frac{d}{d \theta} \sin \theta \frac{d}{d \theta} \tau_{i}=\sigma T_{c}^{4}-\alpha_{i} f \sin \theta
$$

Taking into account the boundary conditions Eq. (34) and Eq. (35), one has

$k_{1} \sin \theta \frac{d}{d \theta} \tau_{1}=\int_{0}^{\theta}\left(\sigma T_{c}^{4}-\alpha_{1} f \sin x\right) \sin x d x$,

$k_{2} \sin \theta \frac{d}{d \theta} \tau_{2}=\int_{\pi / 2}^{\theta}\left(\sigma T_{c}^{4}-\alpha_{2} f \sin x\right) \sin x d x$

The boundary conditions at the ice-water interface give

$\int_{0}^{\theta_{p}}\left(\sigma T_{c}^{4}-\alpha_{1} f \sin x\right) \sin x d x$
$=\int_{\pi / 2}^{\theta_{p}}\left(\sigma T_{c}^{4}-\alpha_{2} f \sin x\right) \sin x d x$

which is equivalent to Eq. (42). Integrating Eqs. (58) and (59) further and taking into account $T=T_{c}$ at $\theta=\theta_{p}$, one has

$$
\begin{aligned}
& T_{1}=T_{c}+\int_{\theta_{p}}^{\theta} \frac{1}{k_{1} \sin y} \int_{0}^{y}\left(\sigma T_{c}^{4}-\alpha_{1} f \sin x\right) \sin x d x, \\
& T_{2}=T_{c}+\int_{\theta_{p}}^{\theta} \frac{1}{k_{2} \sin y} \int_{\pi / 2}^{y}\left(\sigma T_{c}^{4}-\alpha_{2} f \sin x\right) \sin x d x .
\end{aligned}
$$

Respectively,

$$
\begin{aligned}
& \frac{\delta T_{1}}{\delta \theta_{p}}=-\frac{1}{k_{1} \sin \theta_{p}} \int_{0}^{\theta_{p}}\left(\sigma T_{c}^{4}-\alpha_{1} f \sin x\right) \sin x d x, \\
& \frac{\delta T_{2}}{\delta \theta_{p}}=-\frac{1}{k_{2} \sin \theta_{p}} \int_{\pi / 2}^{\theta_{p}}\left(\sigma T_{c}^{4}-\alpha_{2} f \sin x\right) \sin x d x
\end{aligned}
$$

and do not depend on $\theta$.

In the equilibrium one has

$$
\begin{aligned}
& \int_{0}^{\theta_{p}}\left(\sigma T_{1}^{4}-\alpha_{1} f \sin \theta\right) \sin \theta d \theta \\
& +\int_{\theta_{p}}^{\pi / 2}\left(\sigma T_{2}^{4}-\alpha_{2} f \sin \theta\right) \sin \theta d \theta=0
\end{aligned}
$$

which gives in the lowest order

$$
\begin{aligned}
\chi_{p} & =\int_{0}^{\theta_{p}}\left(\sigma T_{c}^{4}-\alpha_{1} f \sin \theta\right) \sin \theta d \theta \\
& =\int_{\pi / 2}^{\theta_{p}}\left(\sigma T_{c}^{4}-\alpha_{2} f \sin \theta\right) \sin \theta d \theta>0,
\end{aligned}
$$


and

$\frac{\delta T_{1}}{\delta \theta_{p}}=-\frac{\chi_{p}}{k_{1} \sin \theta_{p}}<0$,

$\frac{\delta T_{2}}{\delta \theta_{p}}=-\frac{\chi_{p}}{k_{2} \sin \theta_{p}}<0$

Eventually,

$$
\begin{aligned}
\frac{\delta \dot{Q}_{r}}{\delta \theta_{p}} & =-f\left(\alpha_{2}-\alpha_{1}\right) \sin ^{2} \theta_{p} \\
& +4 \sigma T_{c}^{3} \chi_{p}\left(\frac{1-\cos \theta_{p}}{k_{1} \sin \theta_{p}}+\frac{\cos \theta_{p}}{k_{2} \sin \theta_{p}}\right)
\end{aligned}
$$

The obtained relation shows that temperature inhomogeneity improves stability, since terms $\propto 1 / k$ are positive while the instability causing term $\propto\left(\alpha_{2}-\alpha_{1}\right)$ is negative. We have seen earlier that in the case $k \rightarrow \infty$ the mixed state is unstable, while for $k=0$ it is stable. The transition occurs when

$$
\begin{aligned}
& f\left(\alpha_{2}-\alpha_{1}\right) \sin ^{2} \theta_{p} \\
& =4 \sigma T_{c}^{3} \chi_{p}\left(\frac{1-\cos \theta_{p}}{k_{1} \sin \theta_{p}}+\frac{\cos \theta_{p}}{k_{2} \sin \theta_{p}}\right)
\end{aligned}
$$

Since $\chi_{p}$ and $\theta+p$ depend on $f$ the derived condition for the change of stability is a very nonlinear condition on $f$. Further analysis is beyond the framework of the present paper and will be published elsewhere.

\section{Discussion and conclusions}

It is quite clear that the proposed model cannot properly describe the Earth climate. Among the features which have to be included in a realistic model are the multi-layer atmosphere-ocean-solid surface structure, ocean currents and winds, differential volume absorption of the solar radiation, effects of chemical composition, etc. The mentioned factors make the temperature distribution a three-dimensional field which varies at the spatial scales much smaller than the Earth radius and at the temporal scales substantially smaller than the planet rotation period. It is obvious that a simple model like ours cannot possible take into account the small scale fluctuations in a number of parameters. Nevertheless, it is reasonable to expect that the average heat flux in a layer about the surface would be directed from the regions getting more external heat to the regions with weaker heating. In this case, it makes sense to approximate such flux by a gradient like term, similar to the heat conductivity in our model. The highly fluctuating (because of the temperature inhomogeneities and day-night asymmetry) emission from the planet surface may be represented, in the lowest order, by a nonlinear function of the temperature averaged over the small spatial and temporal scales. While this function is not necessarily $T^{4}$ it can be expected to have similar behavior. Thus, we expect that the proposed model does provide information about basic features of the planet climate.
In the above analysis we have shown that the climate of the planet covered by a single phase of a thermally conducting substance is stable, in the sense that small variations of the external energy input results in small variations of the planet temperature. However, when the substance can exist in two different phases, the situation changes drastically. There exists a range of parameters for which the planet is in an unstable state, when a small and slow variation of the solar flux may result in a large global response of the planet. In this case the typical time of the changes on the planet surface depends on the internal planetary parameters and not on the temporal scale of the solar irradiance variations. Simply speaking, when the solar constant drops below some threshold and ice age develops quickly (we warn the reader to not take this words as a direct application to the Earth ice ages). The equilibrium state of the planet becomes history dependent and exhibits a hysteresis behavior. The unstable behavior of the climate and rapid transitions following long periods of slow adiabatic variations resembles the DansgaardOeschger events. The bistable regime and bifurcations of the Stommel type are also the intrinsic features of the proposed model, albeit at this stage verified only for the limiting cases.

To summarize, we have developed a simple lowdimensional model of the planet climate from the first physical principles, not invoking any heuristic ingredients. We have shown that the climate of a two-phase planet is intrinsically unstable. Slow and weak variations of the forcing trigger fast and large changes of the climate, like drastic increase or decrease of the surface area covered with ice. We suggest that this intrinsic instability is the basic feature of most, if not all, climate systems.

Edited by: L. Zelenyi

Reviewed by: N. M. Astafyeva and another anonymous referee

\section{References}

Alley, R. B., Marotzke, J., Nordhauset, W. D., et al.: Abrupt climate change, Science, 299, 2005-2010, 2003.

Boer, G. J., Hamilton, K., and Zhu, W., Climate sensitivity and climate change under strong forcing, Clim. Dynam., 24, 685-700, 2005.

Braun, H., Christl, M., Rahmstorf, S., et al.: Possible solar origin of the 1,470-year glacial climate cycle demonstrated in a coupled model, Nature, 438, 208-211, 2005.

Braun, H., Ganopolski, A., Christl, M., and Chialvo, D. R.: A simple conceptual model of abrupt glacial climate events, Nonlin. Processes Geophys., 14, 709-721, 2007, http://www.nonlin-processes-geophys.net/14/709/2007/.

Budyko,M. I.: The effect of solar radiation variations on the climate of the earth, Tellus, 21, 611-619, 1969.

Cahalan, R. F. and North, G. R.: A Stability Theorem for EnergyBalance Climate Models, J. Atmos. Sci., 36, 1178-1188, 1979.

Cai, M.: Dynamical amplification of polar warming, Geophys. Res. Lett., 32, L22710, doi:10.1029/2005GL024481, 2005. 
Claussen, M., Mysak, L. A., Weaver, A. J., et al.: Earth system models of intermediate complexity: closing the gap in the spectrum of climate system models, Clim. Dynam., 18, 579-586, 2002.

Dansgaard, W., Clausen, H. B., Gundestrup, N., et al.: A New Greenland Deep Ice Core, Science, 218, 1273-1277, 1982.

Galleé, H., van Ypersele, J. P., Fichefet, T., Tricot, C., and Berger, A.: Simulation of the last glacial cycle by a coupled, sectorially averaged climate - ice sheet model, 1 , The climate model, J. Geophys. Res., 96, 13 139-13 161, 1991.

Ganopolski, A., Rahmstorf, S., Petoukhov, V., and Claussen, M.: Simulation of modern and glacial climates with a coupled global model of intermediate complexity, Nature, 391, 351-356, 1998.

Ganopolski, A., and Rahmstorf, S.: Rapid changes of glacial climate simu- lated in a coupled climate model, Nature, 409, 153158, 2001.

Hansen, J., Sato, M., Ruedy, R., et al.: Efficacy of climate forcing, J. Geophys. Res., 110, D18104, doi:10.1029/2005JD005776, 2005.

Lorenz, E. N.: Deterministic nonperiodic flow, J. Atmos. Sci. 20, 130-141, 1963.

North, G. R., Cahalan, R. F., and Coakley, Jr., J. A.: Energy Balance Climate Models (Paper 80R1502), Rev. Geophys. Space Phys., 19, 91-121, 1981.

North, G. R.: The Small Ice Cap Instability in Diffusive Climate Models, J. Atmos. Sci., 41, 3390-3395, 1984.

North, G. R. and Stevens, M. J.: Energy-balance climate models, in: Frontiers of Climate Modeling, edited by: Kiehl, J. T. and Ramanahan, V., Cambridge University Press, Cambridge, 52-82, 2006.

Oerlemans, J.: Glacial cycles and ice-sheet modelling, Clim. Change, 4, 353-374, 1982.
Paillard, D.: Glacial cycles: toward a new paradigm, Rev. Geophys., 39, 325-346, 2001.

Petoukhov, V., Ganopolski, A., Brovkin, V., et al.: CLIMBER-2: a climate system model of intermediate complexity. Part I: Model description and performance for present climate, Clim. Dynam., $16,1-17,2000$.

Pollard, D.: A coupled climate ice sheet model applied to the Quaternary ice ages, J. Geophys. Res., 88, 7705-7718, 1983.

Räisänen, J.: How reliable are climate models?, Tellus A, 59, 1-29, 2007.

Rahmstorf, S.: Abrupt climate change, in: Encyclopedia of Ocean Sciences, edited by: Steele, J., Thorpe, S., and Turekian, K., Academic, London, 1-6, 2001.

Rahmstorf, S.: Ocean circulation and climate during the past 120,000 years, Nature, 419, 207-214, 2002.

Sellers, W. D.: A Global Climatic Model Based on the Energy Balance of the Earth-Atmosphere System, J. Appl. Meteorol., 8, 392-400, 1969.

Stommel, H.: Thermohaline convection with two stable regimes of flow, Tellus, 13 224-13 230, 1961.

Swindles, G. T., Plunkett, G., and Roe, H. M.: A delayed climatic response to solar forcing at $2800 \mathrm{cal}$. BP: multiproxy evidence from three Irish peatlands, The Holocene, 17, 177-182, 2007.

Van Geel, B., Raspopovb, O. M., Renssen, H., et al.: The role of solar forcing upon climate change, Quaternary Sci. Rev., 18, 331338, 1999.

Wu, W. and North, G. R.: Thermal decay modes of a 2-D energy balance climate model, Tellus A, 59, 618-626, 2007. 